\title{
Novel Properties of Fuzzy Labeling Graphs
}

\author{
A. Nagoor Gani, ${ }^{1}$ Muhammad Akram, ${ }^{2}$ and D. Rajalaxmi (a) Subahashini ${ }^{3}$ \\ ${ }^{1}$ PG \& Research Department of Mathematics, Jamal Mohamed College, Trichy, India \\ ${ }^{2}$ Department of Mathematics, University of the Punjab, New Campus, P.O. Box No. 54590, Lahore, Pakistan \\ ${ }^{3}$ Department of Mathematics, Saranathan College of Engineering, Tiruchirappalli, Tamil Nadu 620 012, India
}

Correspondence should be addressed to Muhammad Akram; m.akram@pucit.edu.pk

Received 13 May 2014; Accepted 22 June 2014; Published 9 July 2014

Academic Editor: Pierpaolo D’Urso

Copyright (C) 2014 A. Nagoor Gani et al. This is an open access article distributed under the Creative Commons Attribution License, which permits unrestricted use, distribution, and reproduction in any medium, provided the original work is properly cited.

\begin{abstract}
The concepts of fuzzy labeling and fuzzy magic labeling graph are introduced. Fuzzy magic labeling for some graphs like path, cycle, and star graph is defined. It is proved that every fuzzy magic graph is a fuzzy labeling graph, but the converse is not true. We have shown that the removal of a fuzzy bridge from a fuzzy magic cycle with odd nodes reduces the strength of a fuzzy magic cycle. Some properties related to fuzzy bridge and fuzzy cut node have also been discussed.
\end{abstract}

\section{Introduction}

Fuzzy set is a newly emerging mathematical framework to exemplify the phenomenon of uncertainty in real life tribulations. It was introduced by Zadeh in 1965, and the concepts were pioneered by various independent researches, namely, Rosenfeld [1] and Bhutani and Battou [2] during 1970s. Bhattacharya has established the connectivity concepts between fuzzy cut nodes and fuzzy bridges entitled "Some remarks on fuzzy graphs [3]." Several fuzzy analogs of graph theoretic concepts such as paths, cycles, and connectedness were explored by them. There are many problems, which can be solved with the help of the fuzzy graphs.

Though it is very young, it has been growing fast and has numerous applications in various fields. Further, research on fuzzy graphs has been witnessing an exponential growth, both within mathematics and in its applications in science and Technology. A fuzzy graph is the generalization of the crisp graph. Therefore it is natural that many properties are similar to crisp graph and also it deviates at many places.

In crisp graph, a bijection $f: V \cup E \rightarrow N$ that assigns to each vertex and/or edge if $G=(V, E)$, a unique natural number is called a labeling. The concept of magic labeling in crisp graph was motivated by the notion of magic squares in number theory. The notion of magic graph was first introduced by Sunitha and Vijaya Kumar [4] in 1964. He defined a graph to be magic if it has an edge-labeling, within the range of real numbers, such that the sum of the labels around any vertex equals some constant, independent of the choice of vertex. This labeling has been studied by Stewart $[5,6]$ who called the labeling as super magic if the labels are consecutive integers, starting from 1 . Several others have studied this labeling.

Kotzig and Rosa [7] defined a magic labeling to be a total labeling in which the labels are the integers from 1 to $|V(G)|+|E(G)|$. The sum of labels on an edge and its two endpoints is constant. Recently Enomoto et al. [8] introduced the name super edge magic for magic labeling in the sense of Kotzig and Rosa, with the added property that the $v$ vertices receive the smaller labels. Many other researchers have investigated different forms of magic graphs; for example see Avadayappan et al. [9] Ngurah et al. [10], and Trenkler [11].

In this paper, Section 1 contains basic definitions and in Section 2 a new concept of fuzzy labeling and fuzzy magic labeling has been introduced and also fuzzy star graph is defined. In Section 2, fuzzy magic labeling for some graphs like path, cycle, and star is defined. In Section 3, some properties and results with fuzzy bridge and fuzzy cut nodes are discussed. The graphs which are considered in this paper are finite and connected.

We have used standard definitions and terminologies in this paper. For graphs considered in this paper, the readers are referred to [12-19]. 


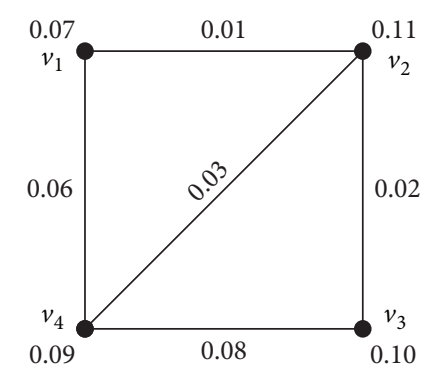

Figure 1: A fuzzy labeling graph.

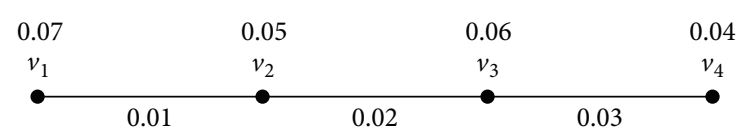

Figure 2: A fuzzy magic path graph: $m_{0}(P)=0.13$.

1.1. Preliminaries. Let $U$ and $V$ be two sets. Then $\rho$ is said to be a fuzzy relation from $U$ into $V$ if $\rho$ is a fuzzy set of $U \times V$. A fuzzy graph $G=(\sigma, \mu)$ is a pair of functions $\sigma: V \rightarrow[0,1]$ and $\mu: V \times V \rightarrow[0,1]$, where for all $u, v \in V$, we have $\mu(u, v) \leq \sigma(u) \wedge \sigma(v)$. A path $P$ in a fuzzy graph is a sequence of distinct nodes $v_{1}, v_{2}, \ldots, v_{n}$ such that $\mu\left(v_{i}, v_{i+1}\right)>0 ; 1 \leq i \leq$ $n$; here $n \geq 1$ is called the length of the path $P$. The consecutive pairs $\left(v_{i}, v_{i+1}\right)$ are called the edge of the path. A path $P$ is called a cycle if $v_{1}=v_{n}$ and $n \geq 3$. The strength of a path $P$ is defined as $\bigwedge_{i=1}^{n} \mu\left(v_{i}, v_{i+1}\right)$. Let $G=(\sigma, \mu)$ be a fuzzy graph. The degree of a vertex $v$ is defined as $d(v)=\sum_{u \neq v, u \in V} \mu(v, u)$. Let $G=(\sigma, \mu)$ be a fuzzy graph. The strong degree of a node $v$ is defined as the sum of membership values of all strong edges incident at $v$. It is denoted by $d_{s}(v)$. Also if $N_{s}(v)$ denote the set of all strong neighbours of $v$, then $d_{s}(v)=\sum_{u \in N_{s}(v)} \mu(v, u)$. An edge is called a fuzzy bridge of $G$ if its removal reduces the strength of connectedness between some pair of nodes in $G$. A node is a fuzzy cut node of $G=(\sigma, \mu)$ if removal of it reduces the strength of connectedness between some other pairs of nodes.

Definition 1 (see, [20]). A graph $G=(\sigma, \mu)$ is said to be a fuzzy labeling graph if $\sigma: V \rightarrow[0,1]$ and $\mu: V \times V \rightarrow[0,1]$ is bijective such that the membership value of edges and vertices are distinct and $\mu(u, v)<\sigma(u) \wedge \sigma(v)$ for all $u, v \in V$.

Example 2 (see, [20]). In Figure $1 \sigma$ and $\mu$ are bijective, such that no vertices and edges receive the same membership value.

Definition 3 (see, [20]). A fuzzy labeling graph is said to be a fuzzy magic graph if $\sigma(u)+\mu(u, v)+\sigma(v)$ has a same magic value for all $u, v \in V$ which is denoted as $m_{0}(G)$.

Example 4 (see, [20]). In Figure $2 \sigma\left(V_{1}\right)+\mu\left(V_{1}, V_{2}\right)+\sigma\left(V_{2}\right)=$ $0.07+0.01+0.05=0.13$, for all $V_{1}, V_{2}, \ldots \in V$.

Definition 5. A star in a fuzzy graph consists of two node sets $V$ and $U$ with $|V|=1$ and $|U|>1$, such that $\mu\left(v, u_{i}\right)>0$ and $\mu\left(u_{i}, u_{i+1}\right)=0,1 \leq i \leq n$. It is denoted by $S_{1, n}$.
Example 6. A fuzzy star graph is shown in Figure 3.

Definition 7 (see, [20]). The fuzzy labeling graph $H=(\tau, \rho)$ is called a fuzzy labeling subgraph of $G=(\sigma, \mu)$ if $\tau(u) \leq \sigma(u)$ for all $u \in V$ and $\rho(u, v) \leq \mu(u, v)$, for all $u, v \in V$.

\section{Properties of Fuzzy Labeling Graphs}

Proposition 8. For all $n \geq 1$, the path $P_{n}$ is a fuzzy magic graph.

Proof. Let $P$ be any path with length $n \geq 1$ and $v_{1}, v_{2}, \ldots, v_{n}$ and $v_{1} v_{2}, v_{2} v_{3}, \ldots, v_{n-1} v_{n}$ are the nodes and edges of $P$. Let $z \rightarrow(0,1]$ such that one can choose $z=0.1$ if $n \leq 4$ and $z=0.01$ if $n \geq 5$. Such fuzzy labeling is defined as follows.

When length is odd:

$$
\begin{gathered}
\sigma^{\omega}\left(v_{2 i-1}\right)=(2 n+2-i) z, \quad 1 \leq i \leq \frac{n+1}{2}, \\
\sigma^{\omega}\left(v_{2 i}\right)=\min \left\{\sigma^{\omega}\left(v_{2 i-1}\right) \mid 1 \leq i \leq \frac{n+1}{2}\right\}-i(z), \\
1 \leq i \leq \frac{n+1}{2}, \\
\mu^{\omega}\left(v_{n-i+2}, v_{n+1-i}\right) \\
=\max \left\{\sigma^{\omega}\left(v_{i}\right) \mid 1 \leq i \leq n+1\right\} \\
-\min \left\{\sigma^{\omega}\left(v_{i}\right) \mid 1 \leq i \leq n+1\right\}-(i-1) z, \\
1 \leq i \leq n .
\end{gathered}
$$

Case (i). $i$ is even.

Then $i=2 x$ for any positive integer $x$ and for each edge $v_{i}, v_{i+1}$

$$
\begin{aligned}
m_{0}(P)= & \sigma^{\omega}\left(v_{i}\right)+\mu^{\omega}\left(v_{i}, v_{i+1}\right)+\sigma^{\omega}\left(v_{i+1}\right) \\
= & \sigma^{\omega}\left(v_{2 x}\right)+\mu^{\omega}\left(v_{2 x}, v_{2 x+1}\right)+\sigma^{\omega}\left(v_{2 x+1}\right) \\
= & \min \left\{\sigma^{\omega}\left(v_{2 i-1}\right) \mid 1 \leq i \leq \frac{n+1}{2}\right\} \\
& -x(z)+\max \left\{\sigma^{\omega}\left(v_{i}\right) \mid 1 \leq i \leq n+1\right\} \\
& -\min \left\{\sigma^{\omega}\left(v_{i}\right) \mid 1 \leq i \leq n+1\right\} \\
& -(n-2 x) z+(2 n-x+1) z \\
= & \min \left\{\sigma^{\omega}\left(v_{2 i-1}\right) \mid 1 \leq i \leq \frac{n+1}{2}\right\} \\
& +\max \left\{\sigma^{\omega}\left(v_{i}\right) \mid 1 \leq i \leq n+1\right\} \\
& -\min \left\{\sigma^{\omega}\left(v_{i}\right) \mid 1 \leq i \leq n+1\right\}+(n+1) z .
\end{aligned}
$$

Case (ii). $i$ is odd.

Then $i=2 x+1$ for any positive integer $x$ and for each edge $v_{i}, v_{i+1}$

$$
\begin{aligned}
m_{0}(P)= & \sigma^{\omega}\left(v_{i}\right)+\mu^{\omega}\left(v_{i}, v_{i+1}\right)+\sigma^{\omega}\left(v_{i+1}\right) \\
= & \sigma^{\omega}\left(v_{2 x+1}\right)+\mu^{\omega}\left(v_{2 x+1}, v_{2 x+2}\right) \\
& +\sigma^{\omega}\left(v_{2 x+2}\right)
\end{aligned}
$$




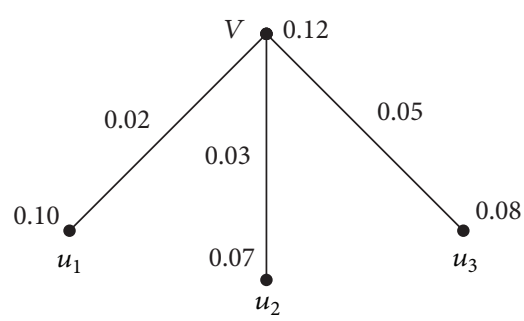

Figure 3: A fuzzy star graph.

$$
\begin{aligned}
= & (2 n-x+1) z \\
& +\max \left\{\sigma^{\omega}\left(v_{i}\right) \mid 1 \leq i \leq n+1\right\} \\
& -\min \left\{\sigma^{\omega}\left(v_{i}\right) \mid 1 \leq i \leq n+1\right\} \\
& -(n-2 x-1) \\
& +\min \left\{\sigma^{\omega}\left(v_{2 i-1}\right) \mid 1 \leq i \leq \frac{n+1}{2}\right\} \\
& -(x+1) z \\
= & \min \left\{\sigma^{\omega}\left(v_{2 i-1}\right) \mid 1 \leq i \leq \frac{n+1}{2}\right\} \\
& +\max \left\{\sigma^{\omega}\left(v_{i}\right) \mid 1 \leq i \leq n+1\right\} \\
& -\min \left\{\sigma^{\omega}\left(v_{i}\right) \mid 1 \leq i \leq n+1\right\} \\
& +(n+1) z .
\end{aligned}
$$

When length is even:

$$
\begin{aligned}
& \sigma^{\omega}\left(v_{2 i}\right)=(2 n+2-i) z, \quad 1 \leq i \leq \frac{n}{2}, \\
& \sigma^{\omega}\left(v_{2 i-1}\right)=\min \left\{\sigma^{\omega}\left(v_{2 i}\right) \mid 1 \leq i \leq \frac{n}{2}\right\}-i(z), \\
& 1 \leq i \leq \frac{n+2}{2}, \\
& \mu^{\omega}\left(v_{n-i+2}, v_{n-i+1}\right)= \max \left\{\sigma^{\omega}\left(v_{i}\right) \mid 1 \leq i \leq n+1\right\} \\
&-\min \left\{\sigma^{\omega}\left(v_{i}\right) \mid 1 \leq i \leq n+1\right\} \\
&-(i-1) z, \quad 1 \leq i \leq n .
\end{aligned}
$$

Case ( $i$ ). $i$ is even.

Then $i=2 x$ for any positive integer $x$ and for each edge $v_{i}, v_{i+1}$

$$
\begin{aligned}
m_{0}(P)= & \sigma^{\omega}\left(v_{i}\right)+\mu^{\omega}\left(v_{i}, v_{i+1}\right)+\sigma^{\omega}\left(v_{i+1}\right) \\
= & \sigma^{\omega}\left(v_{2 x}\right)+\mu^{\omega}\left(v_{2 x}, v_{2 x+1}\right)+\sigma^{\omega}\left(v_{2 x+1}\right) \\
= & (2 n+2-x) z \\
& +\max \left\{\sigma^{\omega}\left(v_{i}\right) \mid 1 \leq i \leq n+1\right\}
\end{aligned}
$$

$$
\begin{aligned}
& -\min \left\{\sigma^{\omega}\left(v_{i}\right) \mid 1 \leq i \leq n+1\right\} \\
& -(n-2 x) z+\min \left\{\sigma^{\omega}\left(v_{2 i}\right) \mid 1 \leq i \leq \frac{n}{2}\right\} \\
& -(x+1) z \\
= & \min \left\{\sigma^{\omega}\left(v_{2 i}\right) \mid 1 \leq i \leq \frac{n}{2}\right\} \\
& +\max \left\{\sigma^{\omega}\left(v_{i}\right) \mid 1 \leq i \leq n+1\right\} \\
& -\min \left\{\sigma^{\omega}\left(v_{i}\right) \mid 1 \leq i \leq n+1\right\}+(n+1) z .
\end{aligned}
$$

Case (ii). $i$ is odd.

Then $i=2 x+1$ for any positive integer $x$ and for each edge $v_{i}, v_{i+1}$

$$
\begin{aligned}
m_{0}(P)= & \sigma^{\omega}\left(v_{i}\right)+\mu^{\omega}\left(v_{i}, v_{i+1}\right)+\sigma^{\omega}\left(v_{i+1}\right) \\
= & \sigma^{\omega}\left(v_{2 x+1}\right)+\mu^{\omega}\left(v_{2 x+1}, v_{2 x+2}\right)+\sigma^{\omega}\left(v_{2 x+2}\right) \\
= & \min \left\{\sigma^{\omega}\left(v_{2 i}\right) \mid 1 \leq i \leq \frac{n}{2}\right\} \\
& -(x+1) z-(n-2 x-1) z \\
& +\max \left\{\sigma^{\omega}\left(v_{i}\right) \mid 1 \leq i \leq n+1\right\} \\
& -\min \left\{\sigma^{\omega}\left(v_{i}\right) \mid 1 \leq i \leq n+1\right\} \\
& +(2 n-x+1) z \\
= & \min \left\{\sigma^{\omega}\left(v_{2 i}\right) \mid 1 \leq i \leq \frac{n}{2}\right\} \\
& +\max \left\{\sigma^{\omega}\left(v_{i}\right) \mid 1 \leq i \leq n+1\right\} \\
& -\min \left\{\sigma^{\omega}\left(v_{i}\right) \mid 1 \leq i \leq n+1\right\}+(n+1) z .
\end{aligned}
$$

Therefore in both the cases the magic value $m_{0}(P)$ is same and unique. Thus $P_{n}$ is fuzzy magic graph for all $n \geq 1$.

Proposition 9. If $n$ is odd, then the cycle $C_{n}$ is a fuzzy magic graph.

Proof. Let $C_{n}$ be any cycle with odd number of nodes and $v_{1}, v_{2}, \ldots, v_{n}$ and $v_{1} v_{2}, v_{2} v_{3}, \ldots, v_{n} v_{1}$ be the nodes and edges of $C_{n}$. Let $z \rightarrow(0,1]$ such that one can choose $z=0.1$ if $n \leq 3$ and $z=0.01$ if $n \geq 4$. The fuzzy labeling for cycle is defined as follows:

$$
\begin{gathered}
\sigma^{\omega}\left(v_{2 i}\right)=(2 n+1-i) z, \quad 1 \leq i \leq \frac{n-1}{2}, \\
\sigma^{\omega}\left(v_{2 i-1}\right)=\min \left\{\sigma^{\omega}\left(v_{2 i}\right) \mid 1 \leq i \leq \frac{n-1}{2}\right\}-i(z), \\
1 \leq i \leq \frac{n+1}{2}, \\
\mu^{\omega}\left(v_{1}, v_{n}\right)=\frac{1}{2} \max \left\{\sigma^{\omega}\left(v_{i}\right) \mid 1 \leq i \leq n\right\}, \\
\mu^{\omega}\left(v_{n-i+1}, v_{n-i}\right)=\mu^{\omega}\left(v_{1}, v_{n}\right)-i(z), \\
1 \leq i \leq n-1 .
\end{gathered}
$$


Case ( $i$ ). $i$ is even.

Then $i=2 x$ for any positive integer $x$ and for each edge $v_{i}, v_{i+1}$

$$
\begin{aligned}
m_{0}\left(C_{n}\right)= & \sigma^{\omega}\left(v_{i}\right)+\mu^{\omega}\left(v_{i}, v_{i+1}\right)+\sigma^{\omega}\left(v_{i+1}\right) \\
= & \sigma^{\omega}\left(v_{2 x}\right)+\mu^{\omega}\left(v_{2 x}, v_{2 x+1}\right) \\
& +\sigma^{\omega}\left(v_{2 x+1}\right) \\
= & (2 n+1-x) z+\frac{1}{2} \max \left\{\sigma^{\omega}\left(v_{i}\right) \mid 1 \leq i \leq n\right\} \\
& -(n-2 x) z \\
& +\min \left\{\sigma^{\omega}\left(v_{2 i}\right) \mid 1 \leq i \leq \frac{n-1}{2}\right\} \\
& -(x+1) z \\
= & \frac{1}{2} \max \left\{\sigma^{\omega}\left(v_{i}\right) \mid 1 \leq i \leq n\right\} \\
& +\min \left\{\sigma^{\omega}\left(v_{2 i}\right) \mid 1 \leq i \leq \frac{n-1}{2}\right\}+n(z) .
\end{aligned}
$$

Case (ii). $i$ is odd.

Then $i=2 x+1$ for any positive integer $x$ and for each edge $v_{i}, v_{i+1}$

$$
\begin{aligned}
m_{0}\left(C_{n}\right)= & \sigma^{\omega}\left(v_{i}\right)+\mu^{\omega}\left(v_{i}, v_{i+1}\right)+\sigma^{\omega}\left(v_{i+1}\right) \\
= & \sigma^{\omega}\left(v_{2 x+1}\right)+\mu^{\omega}\left(v_{2 x+1}, v_{2 x+2}\right) \\
& +\sigma^{\omega}\left(v_{2 x+2}\right) \\
= & \min \left\{\sigma^{\omega}\left(v_{2 i}\right) \mid 1 \leq i \leq \frac{n-1}{2}\right\}-(x+1) z \\
& +\frac{1}{2} \max \left\{\sigma^{\omega}\left(v_{i}\right) \mid 1 \leq i \leq n\right\} \\
& -(n-2 x-1) z+(2 n-x) z \\
= & \frac{1}{2} \max \left\{\sigma^{\omega}\left(v_{i}\right) \mid 1 \leq i \leq n\right\} \\
& +\min \left\{\sigma^{\omega}\left(v_{2 i}\right) \mid 1 \leq i \leq \frac{n-1}{2}\right\}+n(z) .
\end{aligned}
$$

Therefore from above cases $C_{n}$ is a fuzzy magic graph if $n$ is odd.

Proposition 10. For any $n \geq 2$, star $S_{1, n}$ is a fuzzy magic graph.

Proof. Let $S_{1, n}$ be a star graph with $v, u_{1}, u_{2}, \ldots, u_{n}$ as nodes and $v u_{1}, v u_{2}, \ldots, v u_{n}$ as edges.

Let $z \rightarrow(0,1]$ such that one can choose $z=0.1$ if $n \leq 4$ and $z=0.01$ if $n \geq 5$. Such a fuzzy labeling is defined as follows:

$$
\begin{aligned}
\sigma^{\omega}\left(u_{i}\right)= & {[2(n+1)-i] z, \quad 1 \leq i \leq n, } \\
\sigma^{\omega}(v)= & \min \left\{\sigma^{\omega}\left(u_{i}\right) \mid 1 \leq i \leq n\right\}-z, \\
\mu^{\omega}\left(v, u_{n-i}\right)= & \max \left\{\sigma^{\omega}\left(u_{i}\right), \sigma^{\omega}(v) \mid 1 \leq i \leq n\right\} \\
& -\min \left\{\sigma^{\omega}\left(u_{i}\right), \sigma^{\omega}(v) \mid 1 \leq i \leq n\right\} \\
& -i(z), \quad 0 \leq i \leq n-1 .
\end{aligned}
$$

Case (i). $i$ is even.

Then $i=2 x$ for any positive integer $x$ and for each edge $v, u_{i}$

$$
\begin{aligned}
m_{0}\left(S_{1, n}\right)= & \sigma^{\omega}(v)+\mu^{\omega}\left(v, u_{i}\right)+\sigma^{\omega}\left(u_{i}\right) \\
= & \sigma^{\omega}(v)+\mu^{\omega}\left(v, u_{2 x}\right)+\sigma^{\omega}\left(u_{2 x}\right) \\
= & \min \left\{\sigma^{\omega}\left(u_{i}\right) \mid 1 \leq i \leq n\right\}-(z) \\
& +\max \left\{\sigma^{\omega}\left(u_{i}\right), \sigma^{\omega}(v) \mid 1 \leq i \leq n\right\} \\
& -\min \left\{\sigma^{\omega}\left(u_{i}\right), \sigma^{\omega}(v) \mid 1 \leq i \leq n\right\} \\
& -(n-2 x) z+[2(n+1)-2 x] z \\
= & \min \left\{\sigma^{\omega}\left(u_{i}\right) \mid 1 \leq i \leq n\right\} \\
& +\max \left\{\sigma^{\omega}\left(u_{i}\right), \sigma^{\omega}(v) \mid 1 \leq i \leq n\right\} \\
& -\min \left\{\sigma^{\omega}\left(u_{i}\right), \sigma^{\omega}(v) \mid 1 \leq i \leq n\right\} \\
& +(n+1) z .
\end{aligned}
$$

Case (ii). $i$ is odd.

Then $i=2 x+1$ for any positive integer $x$ and for each edge $v, u_{i}$

$$
\begin{aligned}
m_{0}\left(S_{1, n}\right)= & \sigma^{\omega}(v)+\mu^{\omega}\left(v, u_{i}\right)+\sigma^{\omega}\left(u_{i}\right) \\
= & \sigma^{\omega}(v)+\mu^{\omega}\left(v, u_{2 x+1}\right)+\sigma^{\omega}\left(u_{2 x+2}\right) \\
= & \min \left\{\sigma^{\omega}\left(u_{i}\right) \mid 1 \leq i \leq n\right\}-z \\
& +\max \left\{\sigma^{\omega}\left(u_{i}\right), \sigma^{\omega}(v) \mid 1 \leq i \leq n\right\} \\
& -\min \left\{\sigma^{\omega}\left(u_{i}\right), \sigma^{\omega}(v) \mid 1 \leq i \leq n\right\} \\
& -(n-2 x-1) z+[2(n-x)] z \\
= & \min \left\{\sigma^{\omega}\left(u_{i}\right) \mid 1 \leq i \leq n\right\} \\
& +\max \left\{\sigma^{\omega}\left(u_{i}\right), \sigma^{\omega}(v) \mid 1 \leq i \leq n\right\} \\
& -\min \left\{\sigma^{\omega}\left(u_{i}\right) \sigma^{\omega}(v) \mid 1 \leq i \leq n\right\} \\
& +(n+1) z .
\end{aligned}
$$

From the above cases one can easily verify that all star graphs are fuzzy magic graphs.

Remark 11. One can observe the same labeling holds well if we choose the value of $z$ as $0.03,0.05$, and so forth, for the Propositions 8, 9, and 10.

Remark 12. (1) If $G$ is a fuzzy magic graph, then $d(u) \neq d(v)$ for any pair of nodes $u$ and $v$.

(2) For any fuzzy magic graph, $0 \leq d_{s}(v) \leq d(v)$.

(3) Sum of the degree of all nodes in a fuzzy magic graph is equal to twice the sum of membership values of all edges (i.e., $\left.\sum_{i=1}^{n} d\left(v_{i}\right)=2 \sum_{u \neq v} \mu(u, v)\right)$.

(4) Sum of strong degree of all nodes in a fuzzy magic graph is equal to twice the sum of the membership values of all strong arcs in $G$ (i.e., $\left.\sum_{i=1}^{n} d_{s}\left(v_{i}\right)=2 \sum_{u \in N s(v)} \mu(v, u)\right)$. 


\section{Properties of Fuzzy Magic Graphs}

Proposition 13. Every fuzzy magic graph is a fuzzy labeling graph, but the converse is not true.

Proof. This is immediate from Definition 3.

Proposition 14. For every fuzzy magic graph $G$, there exists at least one fuzzy bridge.

Proof. Let $G$ be a fuzzy magic graph, such that there exists only one edge $\mu(x, y)$ with maximum value, since $\mu$ is bijective. Now we claim that $\mu(x, y)$ is a fuzzy bridge. If we remove the edge $(x, y)$ from $G$, then in its subgraph we have $\mu^{\prime \infty}(x$, $y)<\mu(x, y)$, which implies $(x, y)$ is a fuzzy bridge.

Proposition 15. Removal of a fuzzy cut node from a fuzzy magic path $P$ is also a fuzzy magic graph.

Proof. Let $P$ be any fuzzy magic path with length $n$. Then there must be a fuzzy cut node; if we remove that cut node from $P$ then it either becomes a smaller path or disconnected path, anyway it remains to be a path with odd or even length; by Proposition 8 , it is concluded that removal of a fuzzy cut node from a fuzzy magic path $P$ is also a fuzzy magic graph.

Proposition 16. When $n$ is odd, removal of a fuzzy bridge from a fuzzy magic cycle $C_{n}$ is a fuzzy magic graph.

Proof. Let $C_{n}$ be any fuzzy magic cycle with odd nodes. If we choose any path $(u, v)$ then there must be at least one fuzzy bridge, whose removal from $C_{n}$ will result as a path of odd or even length. By Proposition 8, the removal of a fuzzy bridge from a fuzzy magic cycle $C_{n}$ is also a fuzzy magic graph.

Remark 17. (1) Removal of a fuzzy cut node from the cycle $C_{n}$ is also a fuzzy magic graph.

(2) For all fuzzy magic cycles $C_{n}$ with odd nodes, there exists at least one pair of nodes $u$ and $v$ such that $d_{s}(u)=$ $d_{s}(v)$.

Proposition 18. Removal of a fuzzy bridge from a fuzzy magic cycle $C_{n}$ will reduce the strength of the fuzzy magic cycle $C_{n}$.

Proof. Let $C_{n}$ be a fuzzy magic cycle with odd number of nodes. Now choose any path $(u, v)$ from $C_{n}$, and then it is obvious that there exists at least one fuzzy bridge $(x, y)$. Removal of this fuzzy bridge $(x, y)$ will reduce the strength of connectedness between $u$ and $v$. This implies that the removal of fuzzy bridge from the fuzzy magic cycle $C_{n}$ will reduce its strength.

\section{Concluding Remarks}

Fuzzy graph theory is finding an increasing number of applications in modeling real time systems where the level of information inherent in the system varies with different levels of precision. Fuzzy models are becoming useful because of their aim in reducing the differences between the traditional numerical models used in engineering and sciences and the symbolic models used in expert systems. In this paper, the concept of fuzzy labeling and fuzzy magic labeling graphs has been introduced. We plan to extend our research work to (1) bipolar fuzzy labeling and bipolar fuzzy magic labeling graphs and (2) fuzzy labeling and fuzzy magic labeling hypergraphs.

\section{Conflict of Interests}

The authors declare that they do not have any conflict of interests regarding the publication of this paper.

\section{References}

[1] A. Rosenfeld, "Fuzzy graphs," in Fuzzy Sets and Their Applications, L. A. Zadeh, K. S. Fu, and M. Shimura, Eds., pp. 77-95, Academic Press, New York, NY, USA, 1975.

[2] K. R. Bhutani and A. Battou, "On $M$-strong fuzzy graphs," Information Sciences, vol. 155, no. 1-2, pp. 103-109, 2003.

[3] P. Bhattacharya, "Some remarks on fuzzy graphs," Pattern Recognition Letters, vol. 6, no. 5, pp. 297-302, 1987.

[4] M. S. Sunitha and A. Vijaya Kumar, "Complement of a fuzzy graph," Indian Journal of Pure and Applied Mathematics, vol. 33, no. 9, pp. 1451-1464, 2002.

[5] B. M. Stewart, "Magic graphs," Canadian Journal of Mathematics, vol. 18, pp. 1031-1059, 1966.

[6] B. M. Stewart, "Supermagic complete graphs," Canadian Journal of Mathematics, vol. 9, pp. 427-438, 1966.

[7] A. Kotzig and A. Rosa, "Magic valuations of finite graphs," Canadian Mathematical Bulletin, vol. 13, pp. 451-461, 1970.

[8] H. Enomoto, A. S. Llado, T. Nakamigawa, and G. Ringel, "Super edge-magic graphs," SUT Journal of Mathematics, vol. 34, no. 2, pp. 105-109, 1998.

[9] S. Avadayappan, P. Jeyanthi, and R. Vasuki, "Super magic strength of a graph," Indian Journal of Pure and Applied Mathematics, vol. 32, no. 11, pp. 1621-1630, 2001.

[10] A. A. G. Ngurah, A. N. M. Salman, and L. Susilowati, "Hsupermagic labelings of graphs," Discrete Mathematics, vol. 310, no. 8, pp. 1293-1300, 2010.

[11] M. Trenkler, "Some results on magic graphs," in Graphs and Other Combinatorial Topics, M. Fieldler, Ed., vol. 59 of Texte zur Mathematik Band, pp. 328-332, Teubner, Leipzig, Germany, 1983.

[12] M. Akram, "Bipolar fuzzy graphs," Information Sciences, vol. 181, no. 24, pp. 5548-5564, 2011.

[13] M. Akram and W. A. Dudek, "Interval-valued fuzzy graphs," Computers \& Mathematics with Applications, vol. 61, no. 2, pp. 289-299, 2011.

[14] M. Akram and W. A. Dudek, "Intuitionistic fuzzy hypergraphs with applications," Information Sciences, vol. 218, pp. 182-193, 2013.

[15] J. N. Mordeson and P. S. Nair, Fuzzy Graphs and Fuzzy Hypergraphs, Physica, Heidelberg, Germany, 2000.

[16] A. Nagoor Gani and V. T. Chandrasekaran, A First Look at Fuzzy Graph Theory, Allied Publishers, Chennai, India, 2010.

[17] S. Mathew and M. S. Sunitha, "Types of arcs in a fuzzy graph," Information Sciences, vol. 179, no. 11, pp. 1760-1768, 2009.

[18] S. Mathew and M. S. Sunitha, "Node connectivity and arc connectivity of a fuzzy graph," Information Sciences, vol. 180, no. 4, pp. 519-531, 2010. 
[19] J. A. MacDougall and W. D. Wallis, "Strong edge-magic labelling of a cycle with a chord," The Australasian Journal of Combinatorics, vol. 28, pp. 245-255, 2003.

[20] A. Nagoor Gani and D. Rajalaxmi (a) Subahashini, "Properties of fuzzy labeling graph," Applied Mathematical Sciences, vol. 6, no. 69-72, pp. 3461-3466, 2012. 


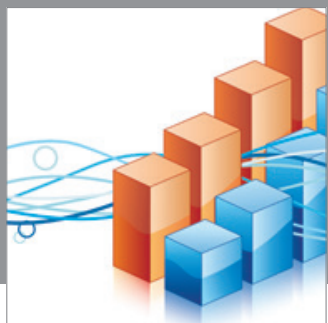

Advances in

Operations Research

mansans

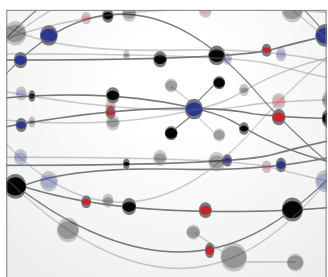

The Scientific World Journal
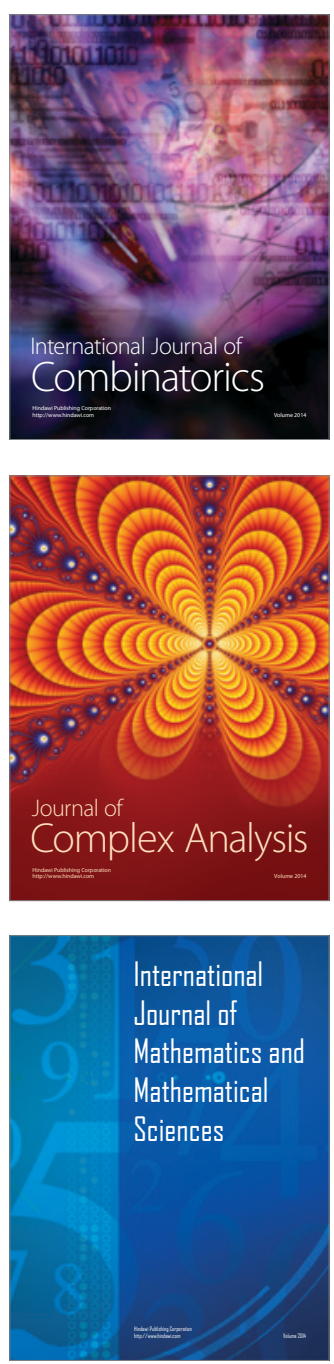
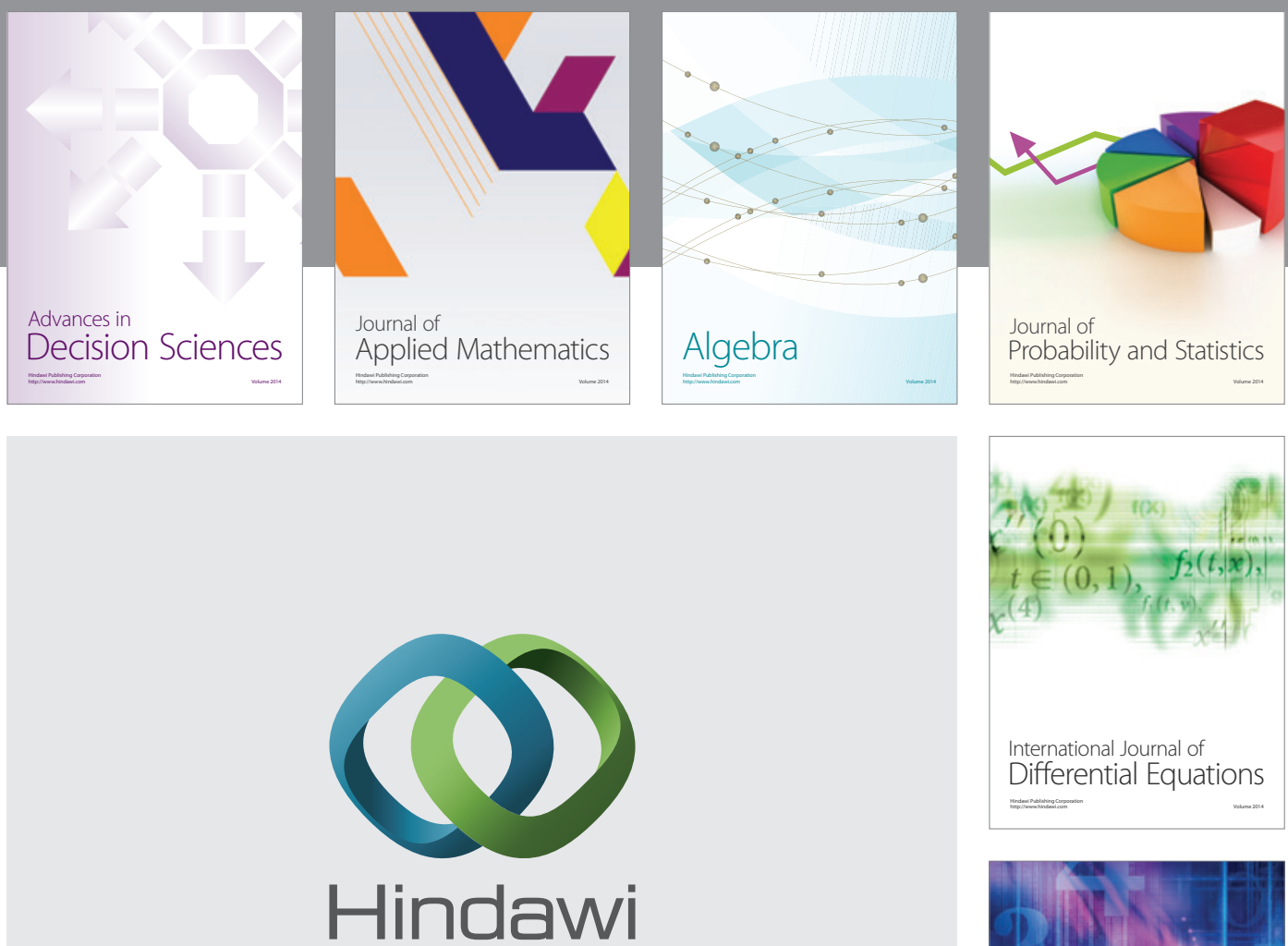

Submit your manuscripts at http://www.hindawi.com
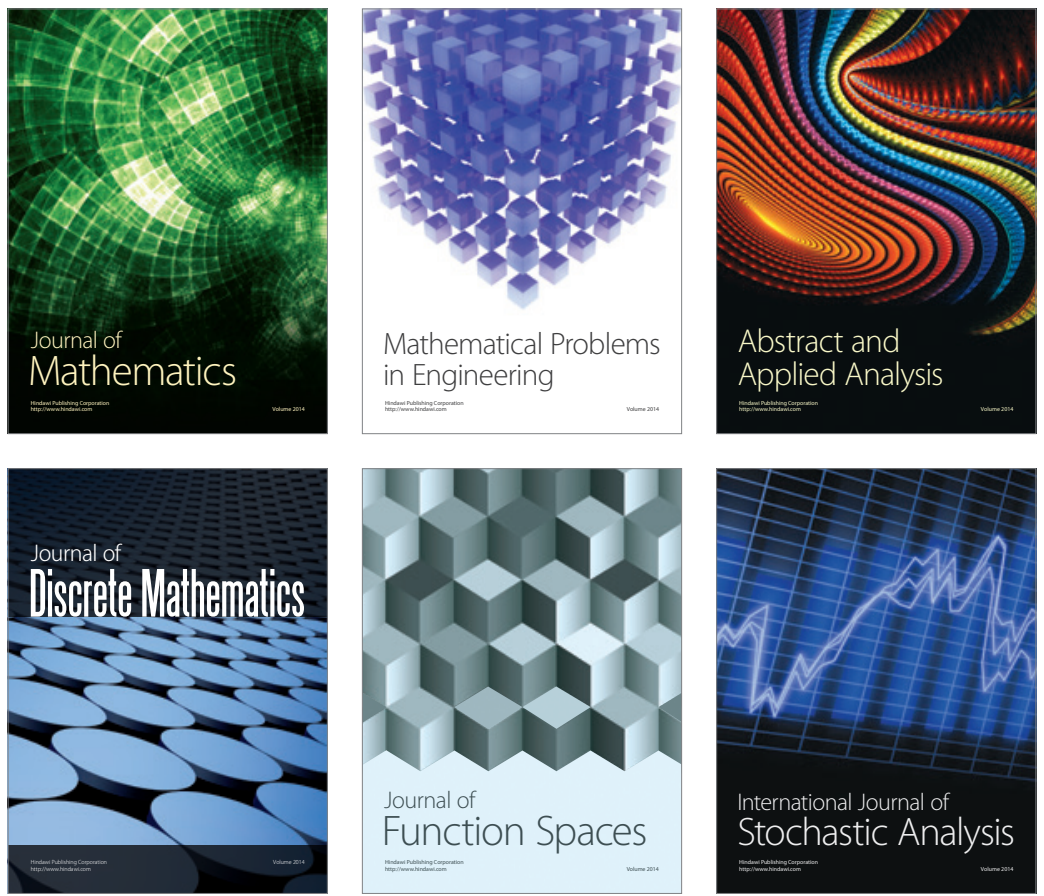

Journal of

Function Spaces

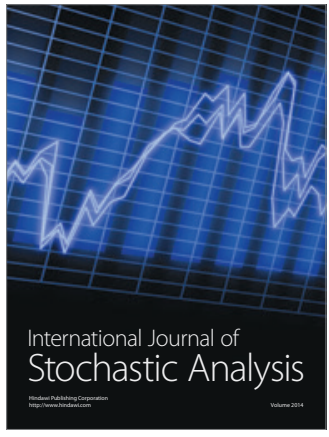

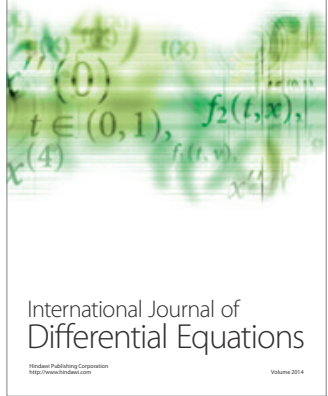
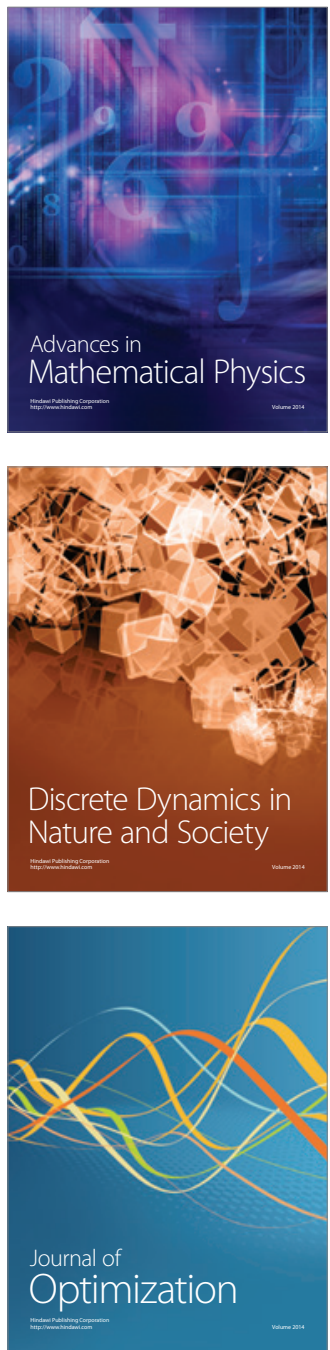